\title{
3'-Daidzein sulfonate sodium inhibits neuronal apoptosis induced by cerebral ischemia-reperfusion
}

\author{
RUIZHEN LIU ${ }^{1,2^{*}}$, XINMING ZHONG ${ }^{2,3^{*}}$, JING ZENG $^{1,2}$, ZHIHUA HUANG $^{1,2}$, \\ $\mathrm{XIAO} \mathrm{LI}^{1,2}, \mathrm{HAI} \mathrm{XIAO}^{1,2}$, QIN CHEN $^{1,2}$ and DONGLIANG LI ${ }^{1,2}$ \\ ${ }^{1}$ Gannan Medical College; ${ }^{2}$ Key Laboratory of Cerebrovascular Diseases of Jiangxi Province; \\ ${ }^{3}$ The First Affiliated Hospital of Gannan Medical University, Ganzhou, Jiangxi 341000, P.R. China
}

Received January 25, 2016; Accepted February 15, 2017

DOI: 10.3892/ijmm.2017.2915

\begin{abstract}
This study aimed to observe the effects of 3'-daidzein sulfonate sodium (DSS) on ischemia-reperfusion-induced brain injury and to analyze the mechanisms responsible for neuronal apoptosis. Focal ischemias were induced in male Sprague-Dawley rats using middle cerebral artery occlusion. The rats were divided into 5 groups based on sham surgery or real occlusion, and treatment with different doses of DSS (0.5, 1.0 and $2.0 \mathrm{mg} / \mathrm{kg}$ ) or normal saline (model group), injected preoperatively into the rats with cerebral occlusion. After $2 \mathrm{~h}$ of ischemia and $24 \mathrm{~h}$ of reperfusion, neurological deficit scores were evaluated using the Longa grade point standard. The infarct volume was measured using a triphenyl tetrazolium chloride staining technique. Blood-brain barrier (BBB) permeability was measured using the Evans blue (EB) content of brain tissues, while electron microscopy was used to observe ultrastructural changes. The expression levels of Bcl-2, Bax and caspase-3 were detected by an immunohistochemical method and western blot analysis. The neurological deficit in rats pre-treated with DSS at all doses decreased significantly $(\mathrm{P}<0.05)$ in comparison with the model group, as did the cerebral infarct volume ratios. The brain EB content was significantly reduced by the injection of DSS. The ultrastructural integrity of the rat BBB was significantly preserved in the DSS-treated groups in comparison with the model group. This was concomitant with the reduced swelling of astrocytes and pericytes in the BBB. The immunohistochemistry results revealed that DSS significantly enhanced the expression of $\mathrm{Bcl}-2$, and inhibited the expression of Bax and caspase- 3 in the brain in comparison to the model group. The number of apoptotic cells in the groups treated with DSS was reduced in comparison with similar areas in the model group. These findings suggest that DSS within a dosage
\end{abstract}

Correspondence to: Dr Jing Zeng, Key Laboratory of Cerebrovascular Diseases of Jiangxi Province, Ganzhou, Jiangxi 341000, P.R. China E-mail: zengjing6168@163.com

*Contributed equally

Key words: 3'-daidzein sulfonate sodium, cerebral ischemia-reperfusion injury, apoptosis, Bcl-2, Bax, caspase-3 range of $0.5-2.0 \mathrm{mg} / \mathrm{kg}$ provides significant protection from injury to the BBB induced by cerebral ischemia-reperfusion, as it exerts a neuroprotective effect by inhibiting apoptosis.

\section{Introduction}

Ischemic cerebral vascular disease is a common disease in clinical neurology, and is caused by problems with blood supply to the brain. Ischemic cerebral vascular disease has high rates of incidence, mortality and recurrence, and is severely damaging to lifestyle and health $(1,2)$. Ultra-early thrombolytic therapy is currently being used to reduce damage to brain tissue in cerebral vascular disease (3); however, the secondary damage caused to the brain following reperfusion has gained much attention (4-7). The exact mechanisms of injury in cerebral ischemia-reperfusion are not yet clear. Findings demonstrate that the possible mechanisms of ischemia-reperfusion injury include the oxygen-derived free radical, nitric oxide (NO), calcium overload in cells, the toxicity of excited amino acids, the inflammatory reaction in local brain tissues and apoptosis (8). Neuronal apoptosis plays an important role in ischemia-reperfusion injury $(9,10)$. There are three types of apoptosis-related genes, including pro-apoptotic genes, antiapoptotic proteins and bidirectional regulator genes. The Bcl-2 family of proteins consists of both anti-apoptotic proteins, such as Bcl-2, and pro-apoptotic proteins such as Bax, which participate in the regulation of apoptosis $(11,12)$. Caspase-3 has also been identified as an enzyme that can trigger a cell apoptotic cascade reaction $(13,14)$.

We previously found that daidzein appeared to have a local anesthetic action (15). It has also been shown to exert protective effects against myocardial ischemia-reperfusion injury in rats following coronary artery lesion (16). Daidzein has an antagonizing role towards $\mathrm{Ca}^{2+}(17)$; it impacts on auto-rhythmicity and contractility of the right atrium in ex vivo rat hearts (18), and it has also been shown to induce the contribution of endothelium-derived hyperpolarizing factor to endothelium relaxation in male rats and in vitro arteries $(19,20)$.

Daidzein is insoluble in water, which results in low oral bioavailability and low efficacy; however, 3'-daidzein sulfonate sodium (DSS) is a newly developed synthetic material with increased water solubility, resulting from modification of the structure of daidzein (an active ingredient of kudzu vine 
root). There are few studies on the pharmacological effects of DSS (21), and the protective effects of DSS against apoptosis in cerebral ischemia have not yet been reported, to the best of our knowledge. Li et al (22) reported that puerarin downregulated caspase-3 protein expression and upregulated Bcl-2 protein expression, which could play a neuroprotective role. However, that study did not consider whether DSS exerts protective effects on cerebral ischemia, or whether it is functionally active in reducing apoptosis.

Thus, in the present study, we aimed to assess the effects of DSS on blood-brain barrier (BBB) permeability, the induction of apoptosis and the expression of Bcl-2, Bax and caspase-3 detected by immunohistochemical methods and western blot analysis.

\section{Materials and methods}

Materials. The Department of Naturally Occurring Drugs and Chemistry, Shenyang Pharmaceutical University (Shenyang, China) provided DSS (C15H907SNa) as a white crystalline powder, with a purity of $>99 \%$. The chemical structures of daidzein and DSS are shown in Fig. 1.

Adult male Sprague-Dawley (SD) rats (250-280 g, $\mathrm{n}=240)$ were purchased from Hunan Silaike Jingda Laboratory Animal Co., Ltd. (Changsha, China). The rats were maintained on a 12-h light and dark cycle, allowed free access to food and water, and allowed to adapt to laboratory conditions for 7 days before the experiment.

All animal experiments were approved by the Animal Care and Use Committee of Gannan Medical College, and conducted according to the National Institutes of Health guidelines.

Preparation of the rat model of cerebral ischemia-reperfusion injury. The rats were anesthetized with $10 \%$ chloral hydrate $(350 \mathrm{mg} / \mathrm{kg}$, intraperitoneally), and a rat model of middle cerebral artery occlusion (MCAO) was established according to the methods of Longa et al (23). A 4-cm long, $0.2-\mathrm{mm}$ in diameter nylon thread was slowly inserted anteriorly towards the direction of the internal carotid artery, through an incision in the common carotid artery trunk. The common carotid artery bifurcation was labeled. After inserting the thread for 18-20 mm, a slight resistance was felt, indicating that the tiny anterior cerebral artery had been reached. The blood supply of the middle cerebral artery was blocked for $1 \mathrm{~h}$. The nylon thread was then pulled out, and the arterial stump was tied. Subcutaneous tissue and skin were sutured. In the sham surgery group, the common carotid artery, external carotid artery, and internal carotid artery were exposed and isolated. The middle cerebral artery was not occluded. During surgery, room temperature was maintained at $23-25^{\circ} \mathrm{C}$.

Experimental grouping. The rats were randomly divided into 5 groups (8 rats per group) as follows: sham surgery, cerebral ischemia-reperfusion injury (model group), lowdose daidzein sulfonate sodium $(0.5 \mathrm{mg} / \mathrm{kg})$, moderate-dose daidzein sulfonate sodium $(1.0 \mathrm{mg} / \mathrm{kg})$ and high-dose daidzein sulfonate sodium $(2.0 \mathrm{mg} / \mathrm{kg})$. Before being reperfused, the different doses of daidzein sulfonate sodium $(0.5,1.0$, $2.0 \mathrm{mg} / \mathrm{kg}$ ) were administered to the drug treatment groups via the sublingual vein. The sham surgery and model groups were administered physiological saline $(0.1 \mathrm{ml} / 100 \mathrm{~g}$ body weight) via the sublingual vein.

Neurological deficit scores. Following $1 \mathrm{~h}$ of ischemia and $24 \mathrm{~h}$ of reperfusion in each group, the degree of damage to the nervous system was evaluated using the Longa grade point standard: a score of 0 indicated no neurologic deficit; a score of 1 (failure to extend left forepaw fully) a mild focal neurologic deficit; a score of 2 (circling to the contralateral side) a moderate focal neurologic deficit; a score of 3 (falling to the contralateral side) a severe neurologic deficit; and a score of 4 was represented by no spontaneous walk and unconsciousness.

Infarct volume measurement. After the neurological deficit tests, rats were decapitated and their brains, including the cerebellum, lower brain stem and olfactory bulb were removed, washed with normal saline (NS), dried with filter paper and frozen. After freezing, the forebrain was generally contained within the thickness of the 5 brain slices cut along the coronal plane. These sections were incubated in $5 \mathrm{ml}$ of $2 \%$ triphenyl tetrazolium chloride solution for $30 \mathrm{~min}$ at $37^{\circ} \mathrm{C}$ in the dark. After staining, non-ischemic regions were colored red, while the infarcted regions were white. The sections were then fixed with $10 \%$ formaldehyde. The infarct volume was calculated using a DT-200 image analysis system (Nanjing Dongtu Technology Co., Ltd, Nanjing, China).

Evaluation of the permeability of the $B B B$. $\mathrm{BBB}$ permeability was measured according to the Evans blue (EB) content in the brain tissues. After infarct modeling (or DSS administration for DSS groups), the rats were immediately injected with $0.25 \mathrm{ml}$ of EB solution ( $0.5 \%$, dissolved in NS) via the tail vein. After $24 \mathrm{~h}$, rats were narcotized and then heart perfused with NS. The forebrain tissue on the ischemic side was then removed, and after weighing, tissues were homogenized in $7.5 \%$ trichloroacetic acid ( $3 \mathrm{ml} / \mathrm{g}$, wet weight). The homogenate was then centrifuged at $12,000 \mathrm{x} \mathrm{g}$ at $4^{\circ} \mathrm{C}$ for $20 \mathrm{~min}$. The optical density (OD) value of the supernatant was read at $620 \mathrm{~nm}$. A calibration curve was set up using a series of EB solution concentrations, with results being indicated by EB brain wet weight $(\mu \mathrm{g})$.

Observation of neurovascular unit ultrastructure by electron microscopy. The rats were narcotized $24 \mathrm{~h}$ after MCAO reperfusion, and then internally fixated with $4 \%$ paraformaldehyde. The parietal cortex brain tissue on the ischemic side was removed and cut into $1 \mathrm{~mm}^{3}$ cubes, fixated for a further $2 \mathrm{~h}$ in a paraformaldehyde-glutaraldehyde solution, and then $2 \mathrm{~h}$ in $1 \%$ osmic acid at $4^{\circ} \mathrm{C}$. After dehydration by methanol gradients, and displacement by epoxypropane, the tissues were embedded and polymerized in polyphenylene sulfide resin. Semi-thin sections were then prepared, stained with methylene blueazure, and observed under an optical lens. Ultra-thin sections were then stained with both uranyl acetate and lead citrate, and observed under an electron microscope (Hitachi H-7650; Hitachi, Tokyo, Japan).

Immunohistochemical detection of Bcl-2, Bax and caspase-3 expression. Following $24 \mathrm{~h}$ of cerebral ischemia-reperfusion, the rats were anesthetized with $10 \%$ chloral hydrate $(3.5 \mathrm{ml} /$ $\mathrm{kg}$, intraperitoneally). A perfusion apparatus filled with $200 \mathrm{ml}$ 
of NS was immediately inserted into the left ventricle via the left ventricular apex, and administration was continued with $300 \mathrm{ml}$ of $4 \%$ paraformaldehyde over a period of $30 \mathrm{~min}$. Brain tissues from the ischemic side were removed and used to prepare paraffin-embedded sections. These sections were incubated in $4 \%$ paraformaldehyde overnight, washed with running water for $24 \mathrm{~h}$, soaked in $50 \%$ ethanol overnight, then stored in $70 \%$ ethanol, followed by $80 \%$ ethanol overnight, followed by soaking in $90 \%$ ethanol for $1 \mathrm{~h}, 95 \%$ ethanol for 45 min (twice), and 100\% ethanol for 45 min (twice). They were then twice-cleared in xylene, for $20 \mathrm{~min}$ each time, before infiltration with wax in a drying machine at a temperature of $60^{\circ} \mathrm{C}$ for $1 \mathrm{~h}$ and $1.5 \mathrm{~h}$, respectively. They were then embedded in paraffin, and the paraffin sections were deparaffinized and cut into $4 \mu \mathrm{m}$ sections, before dewaxing to water. The expression of Bcl-2, Bax and caspase-3 were examined using an immunohistochemistry kit (Booster Bioengineering Institute, Wuhan, China) used according to the manufacturer's instructions. The sections were incubated in $3 \%(\mathrm{v} / \mathrm{v}) \mathrm{H}_{2} \mathrm{O}_{2}$ and washed in distilled water at room temperature for $10 \mathrm{~min}$ ( 3 times) to eliminate endogenous peroxidase activity.

Antigen retrieval protocol. The sections were soaked in $0.01 \mathrm{M}$ citrate buffer solution ( $\mathrm{pH}$ 6.0) and boiled using a pressure cooker. The sections were then rinsed one to two times with phosphate-buffered saline (PBS) solution ( $\mathrm{pH}$ 7.2-7.6), incubated in $1 \%$ bovine serum albumin blocking buffer for $20 \mathrm{~min}$ at room temperature, and excess liquids were then shaken off without further washing. The samples were then incubated with an appropriate diluted primary antibody [Bcl-2 (sc-492; dilution, 1:100; Santa Cruz Biotechnology, Inc., Santa Cruz, CA, USA), Bax (sc-526; dilution, 1:100; Santa Cruz Biotechnology, Inc.); caspase-3 (9664s; dilution, 1:75; Cell Signaling Technology, Inc., Danvers, MA, USA] overnight at $4^{\circ} \mathrm{C}$, before washing in PBS solution ( $\mathrm{pH}$ 7.2-7.6) 3 times, for 2 min each time. They were then incubated for $20 \mathrm{~min}$ at $20-37^{\circ} \mathrm{C}$ with biotinylated goat anti-rabbit IgG (SABC, SA2002, dilution, 1:100; WuHan Boster Biotechnology Limited Company, Wuhan, China), followed by washing in PBS solution ( $\mathrm{pH}$ 7.2-7.6) 3 times, for 2 min each time. The sections were incubated with streptavidin-biotin complex (SABC; SA2002; WuHan Boster Biotechnology Limited Company) at $20-37^{\circ} \mathrm{C}$ for 20 min before washing in PBS solution (pH 7.2-7.6) 4 times, for 5 min each time.

$D A B$ color development. The reagents $\mathrm{A}, \mathrm{B}$ and $\mathrm{C}$ in the DAB color development kit were added to $1 \mathrm{ml}$ distilled water and mixed well. The solution was then added to sections and the reaction was observed under a microscope at room temperature for about $6 \mathrm{~min}$. The sections were then washed in distilled water, stained with hematoxylin, dehydrated, cleared and mounted. Positive cells were identified by yellow-brown granules in the cytoplasm under a light microscope. The percentage of positive neuronal cells was estimated using 10 randomly selected, but representative, high-power visual fields per section.

Western blot analysis of Bcl-2, Bax and caspase-3 protein expression. The total proteins in cerebral ischemia-reperfusion brain were purified, centrifuged, and collected in the supernatant. Proteins were quantified with a bicinchoninic acid assay, separated by sodium dodecyl sulfate-polyacrylamide gel elec-

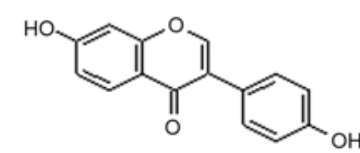

Daidzein

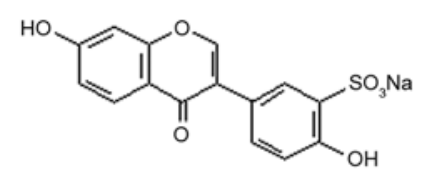

3'-daidzein sulfonate sodium
Figure 1.Chemical structures of daidzein and 3'-daidzein sulfonate sodium(DSS)

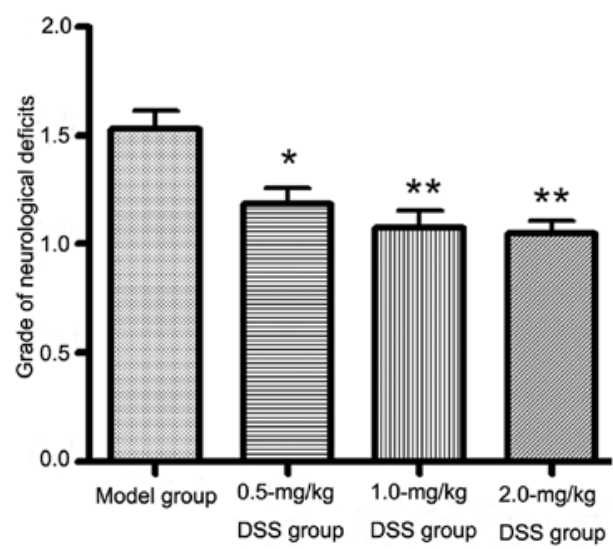

Figure 2. Effects of 3'-daidzein sulfonate sodium (DSS) on neurological deficits. ${ }^{*} \mathrm{P}<0.05 ;{ }^{* *} \mathrm{P}<0.01$ vs. model group.

trophoresis (SDS-PAGE), and transferred onto polyvinylidene fluoride membranes. The membranes were incubated with primary antibodies to Bcl-2 (sc-492; 1:300), Bax (sc-526; 1:300) (both from Santa Cruz Biotechnology, Inc.) and caspase-3 (9664s; 1:500; Cell Signaling Technology, Inc.) overnight at $4^{\circ} \mathrm{C}$. This was followed by incubation with horseradish peroxidase secondary antibodies $(1: 1,000)$ for $2 \mathrm{~h}$ at $37^{\circ} \mathrm{C}$. Images were obtained using a gel image analysis system (Universal Hood II, 721BR04565; Bio-Rad, Hercules, CA, USA) and OD was measured using image lab 3.0 software. The ratio of the OD of the target protein to $\beta$-actin was used to represent the relative expression levels of the target proteins.

Statistical analysis. Data are expressed as the means \pm SEM, and were analyzed using GraphPad Prism version 5.01 software (GraphPad Software, Inc., La Jolla, CA, USA). The group means were compared using one-way analysis of variance (ANOVA) and a Q-test. Comparisons between groups were conducted using paired t-tests. A probability value of $\mathrm{P}<0.05$ was used to determine a significant difference.

\section{Results}

Effects of DSS on neurological deficits. The neurological deficits in the rats pre-treated with DSS $(0.5,1.0$ and $2.0 \mathrm{mg} /$ $\mathrm{kg}$ ) were significantly lower than those in the model group $(\mathrm{P}<0.05$; Fig. 2).

Effects of DSS on cerebral infarction volume. Triphenyl tetrazolium chloride staining revealed that after $24 \mathrm{~h}$ of cerebral ischemia-reperfusion, the infarcted areas of the left cerebral hemisphere, which were mainly in the frontal and parietal cortex, the caudate and the putamen, were white. Normal 

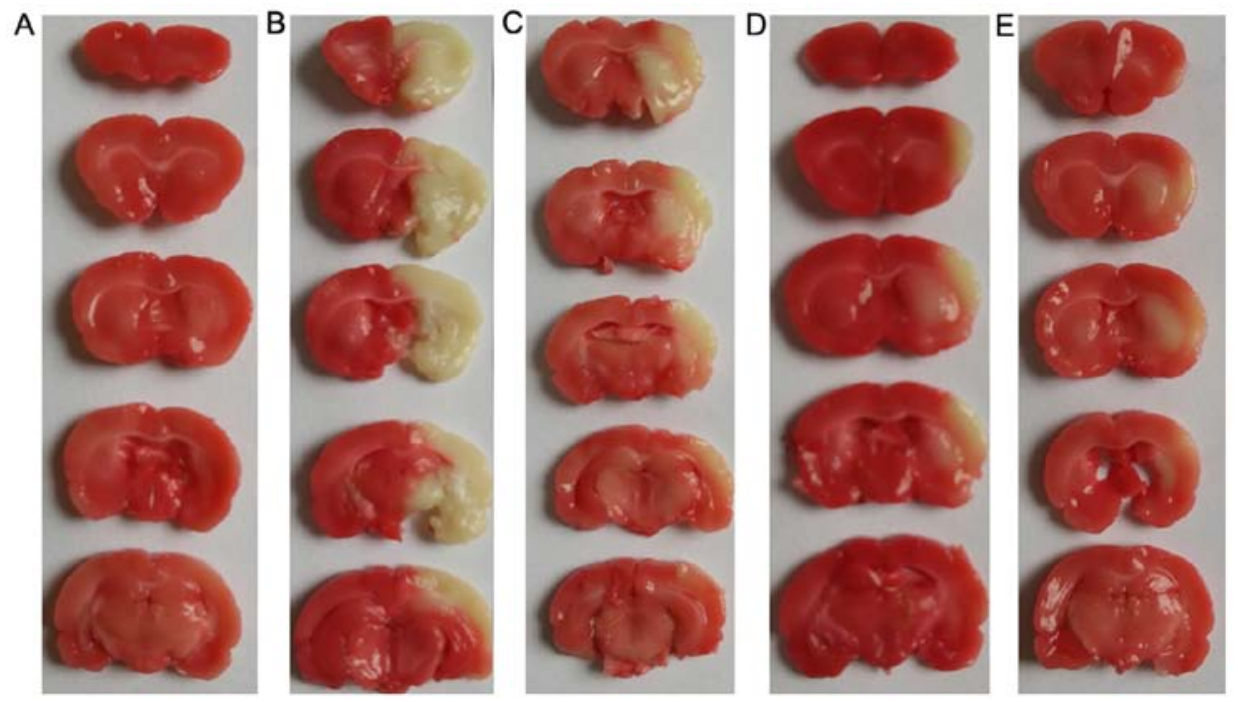

Figure 3 Effects of 3'-daidzein sulfonate sodium (DSS) on cerebral infarction volume. (A) sham-operated group; (B) model group; (C) 0.5-mg/kg DSS-treated group; (D) $1.0-\mathrm{mg} / \mathrm{kg}$ DSS-treated group; and (E) $2.0-\mathrm{mg} / \mathrm{kg}$ DSS-treated group.

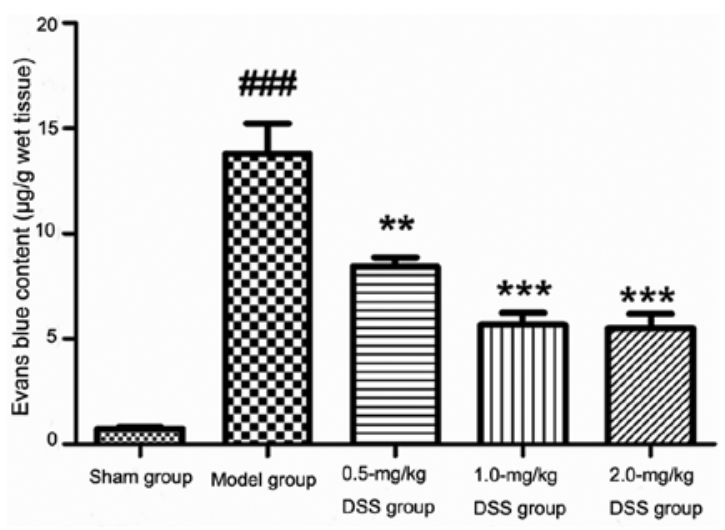

Figure 4. Modulation of blood-brain barrier (BBB) permeability. ${ }^{\# \# \#} \mathrm{P}<0.001$ vs. sham group; ${ }^{* *} \mathrm{P}<0.01 ;{ }^{* * *} \mathrm{P}<0.001$ vs. model group. Sham, sham-operated group.

tissues were stained red, with part of the brain tissue in the penumbral area of the cerebral ischemia-reperfusion injury present as a transition zone of white to red. Compared with the model group, the cerebral infarction volume ratios exhibited obvious reductions in all 3 of the DSS-treated groups (Fig. 3).

Effects of DSS on BBB permeability. As shown in Fig. 4, in comparison with the sham-operated group, BBB permeability and the brain EB content were significantly increased in the model group $(\mathrm{P}<0.001)$. The injection of different doses of DSS $(0.5,1.0$ and $2.0 \mathrm{mg} / \mathrm{kg})$ resulted in a significantly decreased brain EB content in comparison with the model group. This indicated that DSS attenuated the deterioration of BBB permeability in MCAO-induced cerebral ischemiareperfusion injury in rats.

Effects of DSS on the ultrastructure of the BBB. As shown in Fig. 5, in the sham-operated group, the BBB was intact, with intact endothelium cells and a vascular wall structure. The perivascular astrocytic foot processes and pericytes exhibited no swelling, and the vessel lumen was not affected. In the model group, the perivascular astrocytes exhibited obvious swelling, including cytoplasmic vacuolation, edematous fluid, swelling of perivascular foot processes, separation from basement membranes and narrowing of the lumen. Compared with the model group, the swelling of the astrocytes was significantly attenuated in the $0.5-\mathrm{mg} / \mathrm{kg}$ DDS group; the vessel lumen had recovered and blood flow had been restored, although the swelling of the pericytes could still be observed. In the 1.0- and $2.0-\mathrm{mg} / \mathrm{kg}$ DDS groups, the swelling of the pericytes and perivascular astrocytes in the BBB had obviously been inhibited, indicating that DSS attenuated MCAO-induced cerebral ischemia-reperfusion injury in the rat BBB (Fig. 5).

Immunohistochemistry for Bcl-2. Bcl-2-positive yellow-brown granules were observed in the cytoplasm and some nuclei. Bcl-2-positive cells were slightly decreased in number in the model group in comparison with the sham-operated group. Compared with the model group, the number of Bcl-2-positive cells exhibited a significant increase in the DSS-treated groups; the positive cells were also stained more deeply, indicating greater positivity. DSS treatment was shown to upregulate Bcl-2 expression and inhibit apoptosis following cerebral ischemia-reperfusion injury in rats (Fig. 6).

Immunohistochemistry for Bax. Bax-positive yellow-brown granules were located in the cytoplasm and some nuclei. Compared with the sham surgery group, the model group exhibited higher numbers of Bax-positive cells, which indicated that the expression of Bax significantly increased following ischemia-reperfusion injury. The number of Bax-positive cells decreased significantly in the DSS-treated groups compared with the model group. The Bax-positive cells were also more lightly stained, indicating less positivity in the DSS-treated groups. DSS treatment decreased the expression of Bax protein, and inhibited cerebral apoptosis following ischemiareperfusion injury in rats (Fig. 7).

Immunohistochemistry for caspase-3. Caspase-3-positive yellow-brown granules were located in the cytoplasm and 

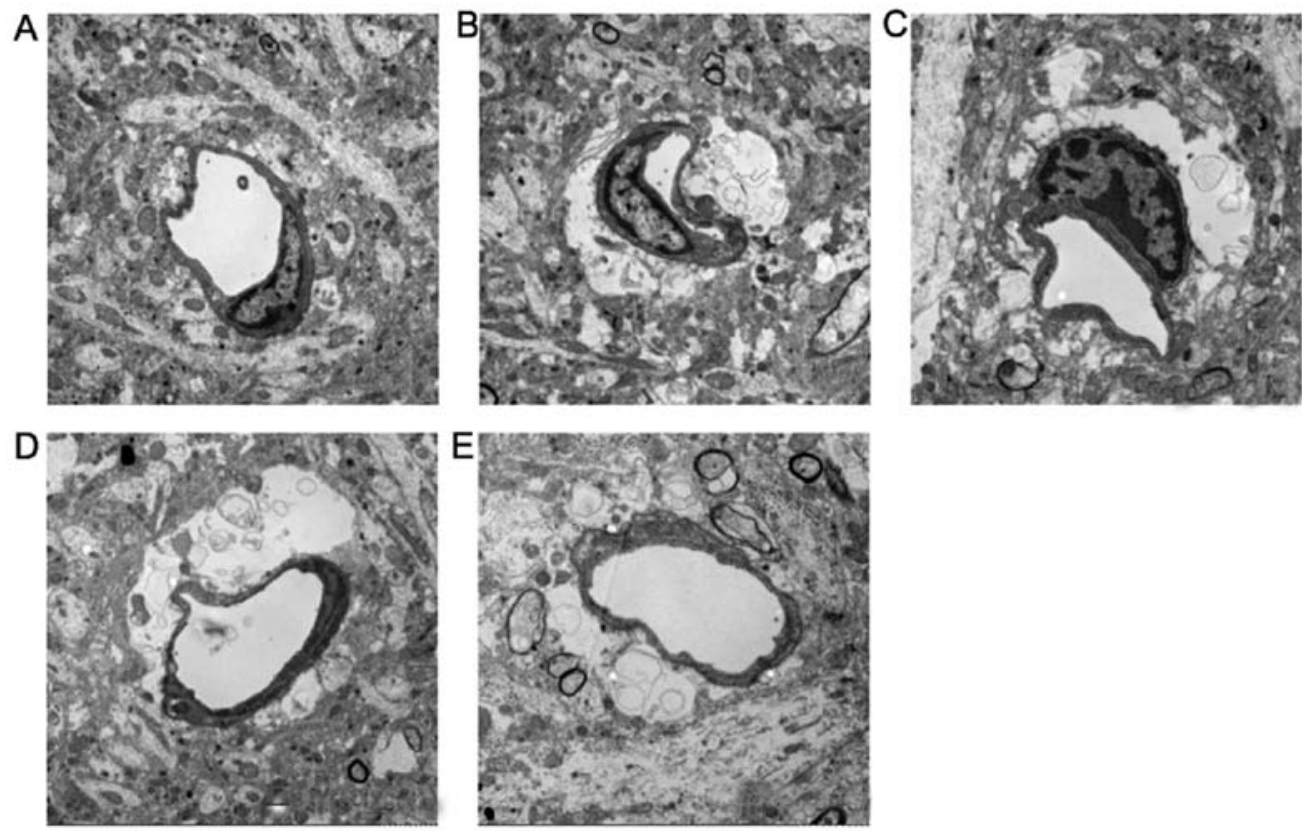

Figure 5. Effects of 3'-daidzein sulfonate sodium (DSS) on the ultrastructure of the blood-brain barrier (BBB). (A) sham-operated group; (B) model group; (C) $0.5-\mathrm{mg} / \mathrm{kg}$ DSS-treated group; (D) 1.0-mg/kg DSS-treated group; and (E) 2.0-mg/kg DSS-treated group.
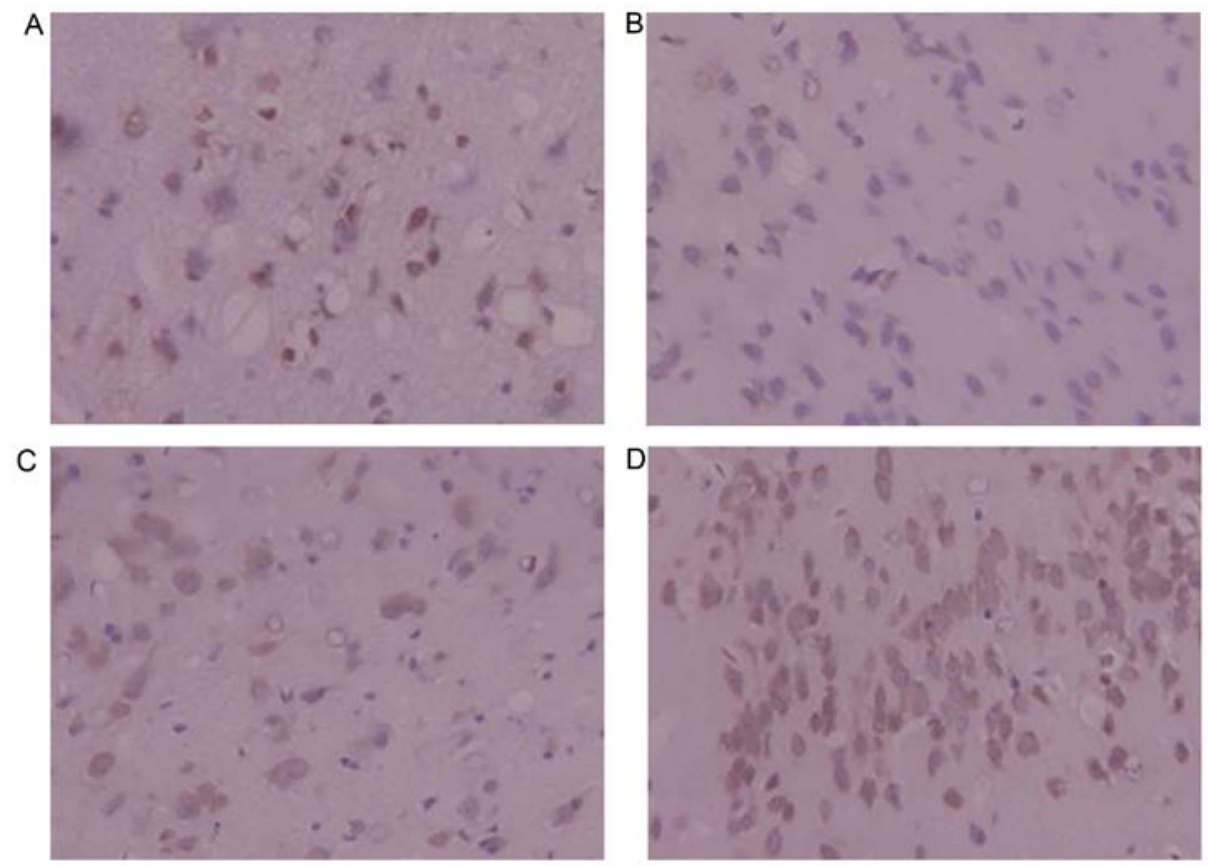

Figure 6. Immunohistochemical staining for Bcl-2 (x40 magnification). (A) sham-operated group; (B) model group; (C) 1.0-mg/kg DSS-treated group; and (D) $2.0-\mathrm{mg} / \mathrm{kg}$ DSS-treated group.

some nuclei. Compared with the sham surgery group, the model group exhibited a large number of caspase-3-positive cells, which indicated that the expression of caspase-3 significantly increased following ischemia-reperfusion injury. The number of caspase-3-positive cells was significantly lower in the DSS-treated groups in comparison with the model group. The positive cells were only lightly stained, indicating that they were only weakly positive. DSS treatment decreased caspase-3 protein expression, and inhibited cerebral apoptosis following ischemia-reperfusion injury in rats (Fig. 8).
Western blot analysis of Bcl-2, Bax and caspase-3

Effect of DSS on Bcl-2 expression. The results of western blot analysis revealed that $\mathrm{Bcl}-2$ expression was significantly reduced in the model group compared with the sham-operated group. A significant increase in Bcl-2 expression was detected in the 1.0- and $2.0-\mathrm{mg} / \mathrm{kg}$ DSS-treated groups, compared with the model group (Fig. 9).

Effect of DSS on Bax expression. The results of western blot analysis revealed that in comparison with the sham-operated group, Bax expression was significantly increased in the model 

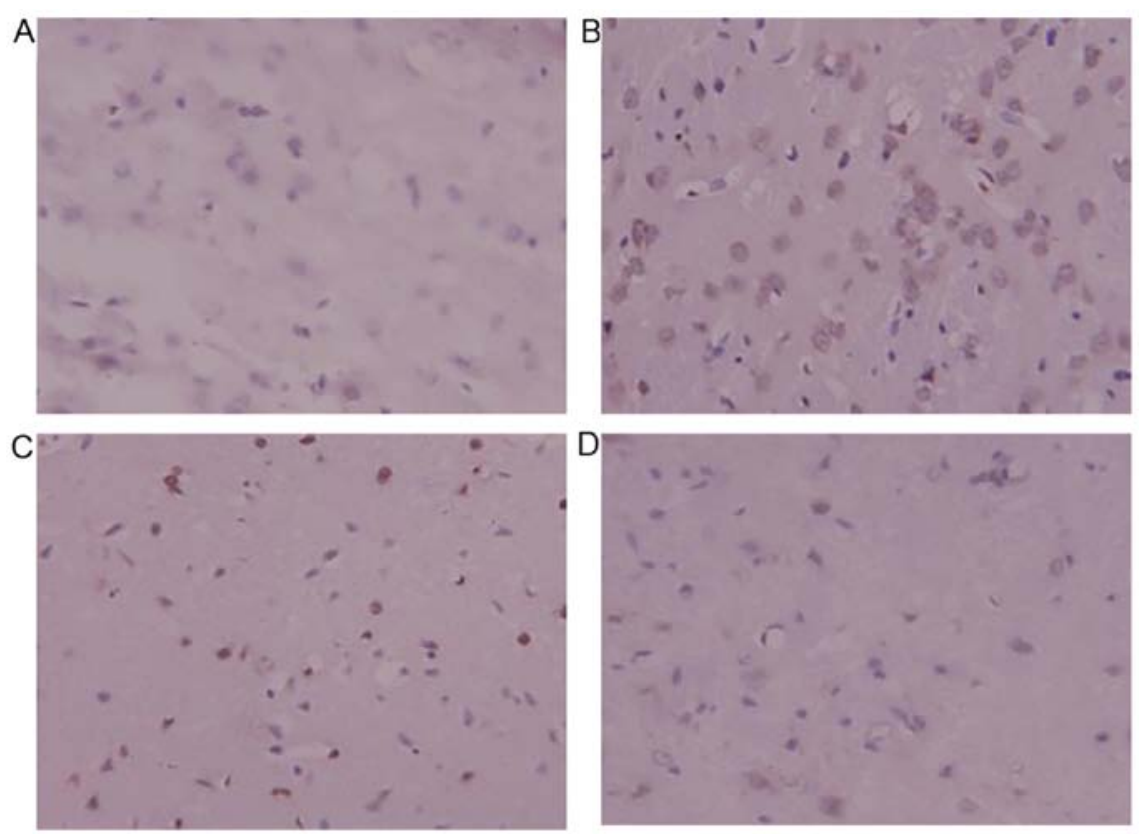

Figure 7. Immunohistochemical for Bax (x40 magnification). (A) sham-operated group; (B) model group; (C) 1.0-mg/kg DSS-treated group; and (D) $2.0-\mathrm{mg} / \mathrm{kg}$ DSS-treated group.
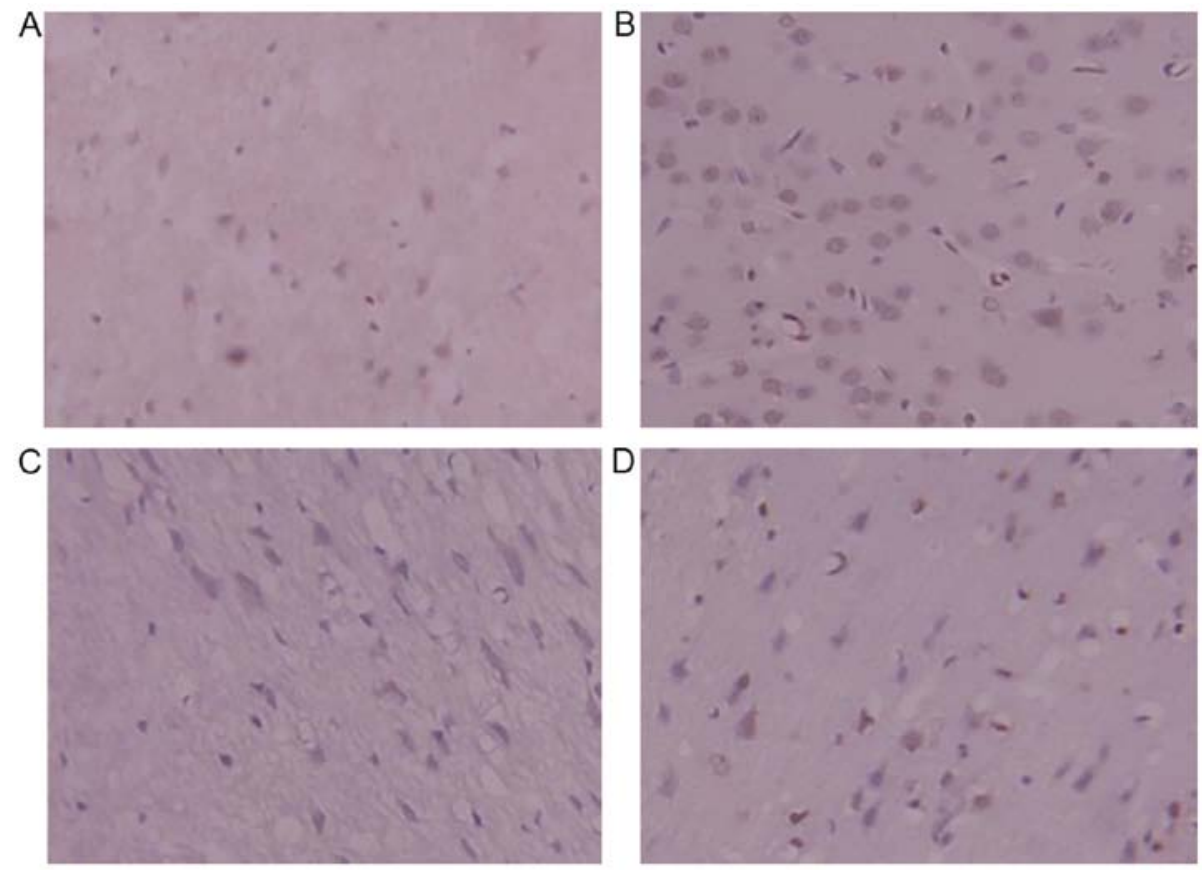

Figure 8. Immunohistochemical for caspase-3 (x40 magnification). (A) sham-operated group; (B) model group; (C) 1.0-mg/kg DSS-treated group; and (D) $2.0-\mathrm{mg} / \mathrm{kg}$ DSS-treated group.

group. A significant decrease in Bax expression was detected in the $1.0-$ and $2.0-\mathrm{mg} / \mathrm{kg}$ DSS-treated groups in comparison with the model group (Fig. 10).

Effect of DSS on caspase-3 expression. The results western blot analysis revealed that in comparison with the sham-operated group, caspase-3 expression was significantly increased in the model group. A significant decrease in caspase-3 expression was detected in the $1.0-$ and $2.0-\mathrm{mg} / \mathrm{kg}$ DSS-treated groups, compared with the model group (Fig. 11).

\section{Discussion}

Ischemia-reperfusion injury is a complex disorder caused by free radicals, NO, calcium overload, excitatory amino acids, inflammation and cell apoptosis. Many drugs have been used in the treatment of cerebral ischemia reperfusion injury, including calcium antagonists, free radical scavengers (24) and growth factors $(25,26)$. Some traditional Chinese medicines have been shown to exert neuroprotective effects on cerebral ischemic 

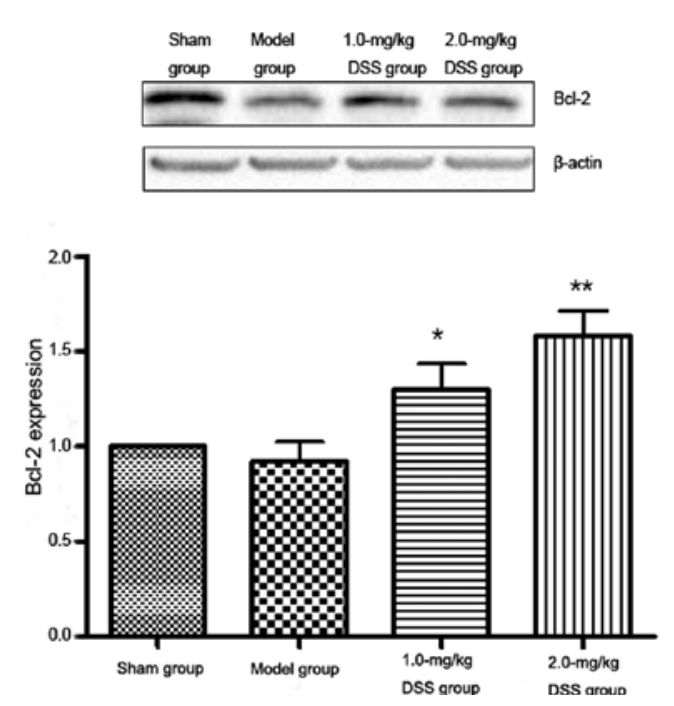

Figure 9. Effect of 3'-daidzein sulfonate sodium (DSS) on Bcl-2 expression during cerebral ischemia-reperfusion injury in rats. ${ }^{*} \mathrm{P}<0.05 ;{ }^{* * *} \mathrm{P}<0.01$ vs. model group. Sham, sham-operated group.

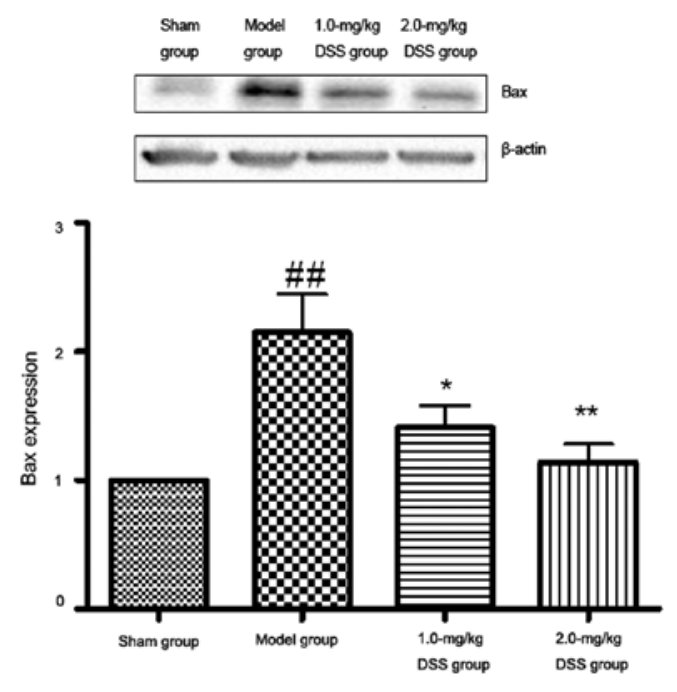

Figure 10. Effect of 3'-daidzein sulfonate sodium (DSS) on Bax expression during cerebral ischemia-reperfusion injury in rats. ${ }^{\# \prime} \mathrm{P}<0.001$ vs. sham group; ${ }^{*} \mathrm{P}<0.05 ;{ }^{* *} \mathrm{P}<0.01$ vs. model group. Sham, sham-operated group.

injury (27-29). It was recently demonstrated (30) that DSS has a low acute toxicity, with the $\mathrm{LD}_{50}$ in rats being $1.75 \mathrm{mg} / \mathrm{kg}$, which is equivalent to 115 -fold the usual daily adult dose. Compared with other medications, DSS is more effective in the treatment of cerebral ischemia-reperfusion injury and is also less toxic; DSS can protect neurons from injury or deterioration.

Cerebral ischemia-reperfusion injury is a complex process involving many factors, with an increase in neurological deficit scores, cerebral blood volume and edema. In the present study, the neurological deficit and the cerebral infarction volume were significantly reduced in the DSS-treated groups $(0.5$, 1.0 and $2.0 \mathrm{mg} / \mathrm{kg})(\mathrm{P}<0.05)$. We also found that DSS can pass through the $\mathrm{BBB}$, relieve perivascular edema of the $\mathrm{BBB}$, maintain the integrity of the vascular wall and reduce ischemiareperfusion-induced damage.

Neuronal apoptosis plays an important role in ischemia-reperfusion injury. Cell apoptosis can be divided into
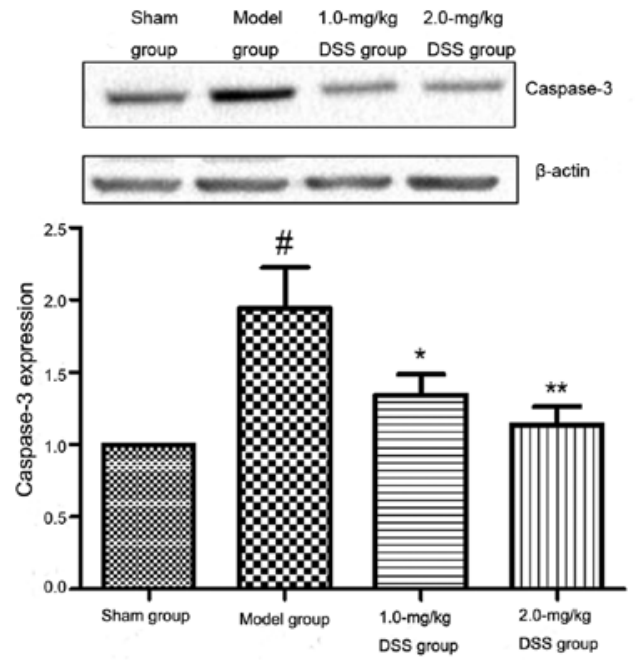

Figure 11. Effect of 3'-daidzein sulfonate sodium (DSS) on caspase-3 expression during cerebral ischemia-reperfusion injury in rats. ${ }^{\#} \mathrm{P}<0.001$ vs. sham group; ${ }^{*} \mathrm{P}<0.05 ;{ }^{* *} \mathrm{P}<0.01$ vs. model group. Sham, sham-operated group.

4 phases: i) apoptotic signal transduction; ii) activation of apoptotic gene expression; iii) triggering of the execution of cell apoptosis; and iv) removal of apoptotic cells. Apoptosisrelated genes can be divided into 3 categories: anti-apoptotic genes, such as Bcl-2; pro-apoptotic genes, such as Bax; and bidirectional regulator genes (31).

$\mathrm{Bcl}-2$ can regulate the activating factor of caspase, inhibit cell damage induced by reactive oxygen species, and change the nuclear-cytoplasmic traffic in cell-cycle regulatory proteins CDK2, CDC2 and p53 (32). The co-expression of Bcl-2 with the gene encoding p53 can delay the apoptosis induced by p53. The synergy between Bcl-2 and Myc prevent movement of p53 into the nucleus, and block p53-induced apoptosis (33). Bcl-2 prevents mitochondrial permeability transition and the release of cytochrome $c$ from mitochondria into the cytoplasm, it inhibits apoptosis by altering the calcium current of intracellular organelles, and promotes the maintenance of calcium homeostasis (34). Bcl-2 can directly combine with inactive CED-4 human homolog Apaf-1 and block caspase (35).

It is known that $\mathrm{Bcl}-2$ and $\mathrm{Bax}$ are apoptosis-regulating proteins that have opposing apoptotic activities. Bcl-2 can form heterodimers with Bax, and the ratio of Bcl-2:Bax could reflect the level of apoptosis; lower Bcl-2:Bax ratios promote apoptosis and higher Bax:Bcl-2 ratios inhibit apoptosis. Bax can induce release of cytochrome $c$, Bax is involved in the regulation pathway of Bcl-xL by combining with it $(36,37)$. In our study, we observed low number of Bcl-2-positive cells in the model group in comparison with the sham-operated group. The number of Bcl-2-positive cells was higher in the DSS-treated groups than in the model group, and a high number of Bax-positive cells was observed in the model group in comparison with the shamoperated group. The number of Bax-positive cells decreased significantly in the DSS-treated groups compared with the model group.

Caspase-3 is a key enzyme that is involved in the execution of apoptosis and leads to disintegration of the cell. It has been reported that caspase- 3 is directly involved in cell apoptosis following cerebral ischemia, by cleaving DNA repair proteins, cytoskeletal proteins, and other related caspase substrate 
proteins, thereby leading to cerebral ischemia reperfusion injury (38). In our study, a large number of caspase-3-positive cells was observed in the model group compared with the sham-operated group. The number of caspase-3-positive cells in the DSS-treated groups decreased significantly compared with that in the model group.

Our findings suggest that DSS at a dose range of $0.5-2.0 \mathrm{mg} / \mathrm{kg}$ can significantly promote the expression of Bcl-2, inhibit the expression of caspase-3 and Bax, and reduce the Bcl-2:Bax ratio. DSS exerts a neuroprotective effect on cerebral ischemia-reperfusion by regulating the expression of $\mathrm{Bcl}-2$, Bax and caspase-3.

\section{Acknowledgements}

This study was supported by grants from the National Natural Science Foundation of China (NSFC; nos. 81160399, 81560583) and from the implement plan of Science and Technology in Universities of Jiangxi Province (Science Frontier) (no. KJLD13085).

\section{References}

1. Ministry of Health of the People's Republic of China: Summary of Chinese health statistics. Beijing: People's Medical Publishing House, 2012.

2. Sauser K, Burke JF, Reeves MJ, Barsan WG and Levine DA: A systematic review and critical appraisal of quality measures for the emergency care of acute ischemic stroke. Ann Emerg Med 64: 235-244, 2014.

3. Fang YL, Luo YM and Zhao YM: Protective effect and the related mechanisms of rhubarb on ischemic cerebrovascular disease. ShouDu Yikedaxue Xuebao 36: 718-722, 2015.

4. Dong GX and Feng YP: Effects of NBP on ATPase and antioxidant enzymes activities and lipid peroxidation in transient focal cerebral ischemic rats. Zhongguo Yi Xue Ke Xue Yuan Xue Bao 24: 93-97, 2002.

5. Liu R, Gao WJ, Qian T and Wang L: Astragalus injection inhibits expression of Apaf-1 in rat hippocampus after cerebral ischemia and reperfusion. Zhongguo Bingli Shengli Zazhi 29: 872-877, 2013.

6. Qu HY and Yuan J: Effects of Ginsenoside Rg1 on nNOS and iNOS expressions in rat brain tissue after cerebral ischemia reperfusion. Tianjin Yiyao 42: 889-892, 2014.

7. Li H, Deng CQ, Chen BY, Chen RF, Zhang SP and Liang Y: Effects of Panax Notoginseng saponins on expression of caspase after focal cerebral ischemia-reperfusion in rats. Chin Pharmacol Bull 22: 189-193, 2006.

8. Li CY and Li WH: Research progress of the mechanisms of cerebral ischemia reperfusion injury. Zhonghua Xiandai Zhongxiyi Zazhi 3: 1744-1746, 2005.

9. Kuschinsky W and Gillardon F: Apoptosis and cerebral ischemia. Cerebrovasc Dis 10: 165-169, 2000.

10. Uchino H, Morota S, Hirabayashi G, Ushijima K, Kakinuma T, Ishii N, Shibasaki F and Kuroda Y: Molecular mechanism of ischemic brain injuries and perspectives of drug therapies for neuroprotection. Masui 56: 248-270, 2007 (In Japanese).

11. Zhuo AS, Chen AJ and Hua WJ: Effects of cerebral ischemia and reperfusion injury on apoptosis of cell and expression of apoptosis gene in the different tissues of rats. Zhongguo Linchuang Kangfu 6: 1263-1264, 2002.

12. Xue Q, Zou YA, Zhao AM and He XF: Effects of Kangnaoye 1 on cerebral ischemia-reperfusion half silent zone apoptosis and $\mathrm{Bcl}-2 / \mathrm{Bax}$ ratio in rats. Zhongguo Quanke Yixue 13: 498-501, 2010.

13. Khalil H, Peltzer N, Walicki J, Yang JY, Dubuis G, Gardiol N, Held W, Bigliardi P, Marsland B, Liaudet L and Widmann C: Caspase-3 protects stressed organs against cell death. Mol Cell Biol 32: 4523-4533, 2012.

14. Yu L, Miao H, Hou Y, Zhang B and Guo L: Neuroprotective effect of A20 on TNF-induced postischemic apoptosis. Neurochem Res 31: 21-32, 2006

15. Wang QH, Li DL and Huang ZH: The anti-oxidation effects of daidzin on isolated myocardial ischemia/reperfusion injury.J Gannan Med Univ 31: 189-191, 2011.
16. Qi JP, Wu AP, Wang DS, Wang LF, Li SX and Xu FL: Correlation between neuronal injury and Caspase-3 after focal ischemia in human hippocampus. Chin Med J (Engl) 117: 1507-1512, 2004.

17. Ye HY, Chen QY, Qiu F, Huang ZH, Huang YP, Xiao H and Zeng J: Effect of daidzein on atrial electrophysiological characteristic in guinea pig. Zhong Yao Cai 29: 312-313, 2006.

18. Ye HY, Hu ZP, Zhou L, Huang ZH and Zeng J: Protective effect of daidzein on myocardial ischemia injury in rats. Pharmacol Clin Chin Mater Med 27: 221-223, 2006.

19. Woodman OL and Boujaoude M: Chronic treatment of male rats with daidzein and 17 beta-oestradiol induces the contribution of EDHF to endothelium-dependent relaxation. Br J Pharmacol 141: 322-328, 2004.

20. Nevala R, Paukku K, Korpela R and Vapaatalo H: Calcium-sensitive potassium channel inhibitors antagonize genistein- and daidzein-induced arterial relaxation in vitro. Life Sci 69: 14071417, 2001.

21. Huang YS, Zeng J, Huang YP, Qiu F, Ye HY and Wang SR: Antagonistic effect of 3'-daidzein sulfonate sodium on prostatic hyperplasia in mice. Zhonghua Nan Ke Xue 13: 387-390, 2007 (In Chinese).

22. Li CT, Wang YL, Wu GT, Cheng XL, She YL, Huang Y and Chen YF: Effect of Puerarin on Caspase-3 and Bcl-2 expression of hippocampal CA1 neurons in ovariectomized rats. Zhongguo Zhongyiyao Xinxi Zazhi 21: 40-46, 2014.

23. Longa EZ, Weinstein PR, Carlson S and Cummins R: Reversible middle cerebral artery occlusion without craniectomy in rats. Stroke 20: 84-91, 1989.

24. Huang HF, Guo F, Cao YZ, Shi W and Xia Q: Neuroprotection by manganese superoxide dismutase (MnSOD) mimics: antioxidant effect and oxidative stress regulation in acute experimental stroke. CNS Neurosci Ther 18: 811-818, 2012.

25. Homi HM, Sheng H, Arepally GM, Mackensen GB and Grocott HP: Aprotinin improves functional outcome but not cerebral infarct size in an experimental model of stroke during cardiopulmonary bypass. Anesth Analg 111: 38-45, 2010.

26. Liu J and Wang LN: Gamma aminobutyric acid (GABA) receptor agonists for acute stroke. Cochrane Database Syst Rev: CD009622, 2014.

27. Lin Z, Zhu D, Yan Y and Yu B: Herbal formula FBD extracts prevented brain injury and inflammation induced by cerebral ischemia-reperfusion. J Ethnopharmacol 118: 140-147, 2008.

28. Chan SJ, Wong WS, Wong PT and Bian JS: Neuroprotective effects of andrographolide in a rat model of permanent cerebral ischaemia. Br J Pharmacol 161: 668-679, 2010.

29. Sun K, Fan J and Han J: Ameliorating effects of traditional Chinese medicine preparation, Chinese materia medica and active compounds on ischemia/reperfusion-induced cerebral microcirculatory disturbances and neuron damage. Acta Pharm Sin B 5: 8-24, 2015.

30. Meng QH: Study on acute toxicity of 3'-daidzein sulfonate sodium in mice. Zhongyiyao Daobao 19: 78-79, 2013.

31. Wang JZ and Yin CH: Pathophysiology. Beijing: People's Medical Publishing House, 2013.

32. He G, Siddik ZH, Huang Z, Wang R, Koomen J, Kobayashi R, Khokhar AR and Kuang J: Induction of p21 by p53 following DNA damage inhibits both Cdk4 and Cdk2 activities. Oncogene 24: 2929-2943, 2005.

33. Goloudina AR, Mazur SJ, Appella E, Garrido C and Demidov ON: Wip1 sensitizes $\mathrm{p} 53$-negative tumors to apoptosis by regulating the Bax/Bcl-xL ratio. Cell Cycle 11: 1883-1887, 2012.

34. Wu C, Fujihara H, Yao J, Qi S, Li H, Shimoji K and Baba H: Different expression patterns of $\mathrm{Bcl}-2, \mathrm{Bcl}-\mathrm{xl}$, and Bax proteins after sublethal forebrain ischemia in C57Black/Crj6 mouse striatum. Stroke 34: 1803-1808, 2003.

35. Inohara N, Gourley TS, Carrio R, Muñiz M, Merino J, Garcia I, Koseki T, Hu Y, Chen S and Núñez G: Diva, a Bcl-2 homologue that binds directly to Apaf-1 and induces BH3-independent cell death. J Biol Chem 273: 32479-32486, 1998.

36. Jin HM: Pathophysiology. People's Medical Publishing House, Beijing, pp178-180, 2000.

37. Behrends M, Martinez-Palli G, Niemann CU, Cohen S, Ramachandran R and Hirose R: Acute hyperglycemia worsens hepatic ischemia/reperfusion injury in rats. J Gastrointest Surg 14: 528-535, 2010.

38. Harrison DC, Davis RP, Bond BC, Campbell CA, James MF, Parsons AA and Philpott KL: Caspase mRNA expression in a rat model of focal cerebral ischemia. Brain Res Mol Brain Res 89: 133-146, 2001. 